\title{
Evaluation of endocan and endoglin levels in chronic kidney disease due to diabetes mellitus
}

\author{
Betül Ekiz-Bilir¹, Bülent Bilir², Murat Aydın², Neslihan Soysal-Atile ${ }^{1}$
}

${ }^{1}$ Endocrinology and Metabolic Diseases Division, Tekirdag State Hospital, Tekirdag,
Turkey
${ }^{2}$ Internal Medicine Department, Medical Faculty, Namik Kemal University, Tekirdağ,
Turkey
${ }^{3}$ Biochemistry Department, Medical Faculty, Namik Kemal University, Tekirdağ, Turkey

Submitted: 12 July 2016

Accepted: 9 November 2016

Arch Med Sci 2019; 15, 1: 86-91

DOI: https://doi.org/10.5114/aoms.2018.79488

Copyright (c) 2018 Termedia \& Banach

\section{Abstract}

Introduction: Endocan and endoglin have been shown to play a role in angiogenesis. Aberrant excessive angiogenesis is a main factor in the development of diabetic nephropathy. In this study we evaluated endocan and endoglin levels in diabetes patients with and without albuminuria and compared them with healthy subjects. Therefore we aimed at gaining a better understanding of the role of angiogenesis in diabetic nephropathy and to assess the predictive role of endocan and endoglin as markers of diabetic nephropathy progression.

Material and methods: Ninety-six type 2 diabetes patients were classified according to their 24-hour urinary albumin excretion rate. Forty type 2 diabetes patients with normoalbuminuria (urinary albumin excretion $<30 \mathrm{mg} /$ day), 56 type 2 diabetes patients with diabetic nephropathy (with a urinary albumin excretion $\geq 30 \mathrm{mg} /$ day) and 35 healthy non-diabetic control subjects were included. Their anthropometric features, arterial blood pressures, fasting glucose, glycated hemoglobin, urea, creatinine, lipids, endocan and endoglin levels were measured and compared to each other.

Results: Endocan and endoglin levels of diabetics patients were higher than those of the controls. In comparison of endocan and endoglin levels of diabetic nephropathy patients with controls, $p$-values were $<0.001$ and 0.002 respectively. In comparison of normoalbuminuric diabetic patients with controls, $p$-values were 0.001 and 0.017 respectively. Endocan levels of diabetic nephropathy cases were higher than those of normoalbuminuric patients $(p=0.011)$ but there was no statistically significant difference in endoglin levels between them $(p=0.822)$.

Conclusions: Endocan might be a more reliable marker of diabetic nephropathy development than endoglin.

Key words: diabetic nephropathy, endocan, endoglin, angiogenesis.

\section{Introduction}

Chronic kidney disease (CKD) is a public health burden that has increased in the last years $[1,2]$. The leading cause of CKD in the world currently is diabetes mellitus (DM). Diabetic nephropathy (DN) is a significant medical problem because of its increasing incidence, morbidity, and mortality. Diabetic nephropathy is a microvascular complication of DM that has been observed in 30-40\% of type 1 DM and $10-20 \%$ of type 2 DM patients $[3,4]$.

\author{
Corresponding author: \\ Betül Ekiz-Bilir \\ Endocrinology and \\ Metabolic Diseases \\ Division \\ Tekirdag State \\ Hospital \\ 59030 Tekirdag, Turkey \\ Phone: + 905057745996 \\ E-mail: \\ bekiz99@yahoo.com.tr
}


Diabetic nephropathy is diagnosed by the help of glomerular filtration rate and albuminuria levels which were confirmed in at least 2 control tests 3-6 months apart [5]. In 1985, at a conference convened to achieve a consensus for definition of microalbuminuria, an albumin excretion rate of 20$200 \mu \mathrm{g} / \mathrm{min}$, which is equivalent to $30-300 \mathrm{mg} /$ day of urinary daily albumin excretion, was recognized as microalbuminuria [6].

The pathophysiology of this glomerulopathy with complex structural, functional and clinical changes over time has not been exactly elucidated yet $[1,3,4]$. The mechanisms of DN pathophysiology are very complex. Hyperglycemia and hemodynamic changes are suggested to act together. One of the principal pathogenic mechanisms of this disease is aberrant excessive angiogenesis. There is increasing evidence that angiogenic growth factors contribute to diabetic nephropathy. Pro-angiogenic and anti-angiogenic factors are normally in a strict balance. But in some pathological conditions like DM, there is an imbalance between them in such a way that pro-angiogenic factors have dominance over down-regulated anti-angiogenic molecules. So this imbalance leads to increased proliferation and migration of endothelial cells and results in immature and leaky vessels $[7,8]$.

Endocan, a $50 \mathrm{kDa}$ soluble proteoglycan, is a novel human endothelial cell specific molecule (endothelial cell specific molecule-1 - ESM-1), secreted by vascular endothelial cells and suggested as an indicator of endothelial cell activation and angiogenesis [9]. It has some role in biological processes such as neovascularization, differentiation, cell adhesion, migration and proliferation [10]. While the endothelium of highly vascularized organs such as brain, heart, liver and pancreas had no detectable expression of endocan, activated endothelium during inflammation and tumor progression shows a several-fold increase in endocan mRNA [11]. Some pro-angiogenic factors, e.g. vascular endothelial growth factor (VEGF) and fibroblast growth factor-2 (FGF-2), stimulate expression of endocan [9]. Endocan is suggested to play a role in the pathogenesis of inflammation, vascular disorders and endothelial dysfunction. Endocan levels are elevated in chronic kidney disease, renal transplant rejection, tumor progression and hypertension [12].

Endoglin (CD105) is a type-III transmembrane glycoprotein that acts as an auxiliary receptor for transforming growth factor $\beta$ (TGF- $\beta$ ) [13]. It is not an actual receptor for TGF- $\beta$ but it strongly modulates the phosphorylation process of TGF- $\beta$ RII. It inhibits the TGF- $\beta$ signaling pathway and endothelial nitric oxide synthase expression. It is known as a hypoxiainducible glycoprotein. Even though endoglin is predominantly expressed in proliferating endothelial cells in the vessel wall, it is also expressed by mono- cytes/macrophages, fibroblasts and vascular smooth muscle cells [14]. Endoglin is required for normal angiogenesis so its expression is up-regulated in developing embryos and during wound healing, inflammation, atherosclerosis, hypoxia, vascular injury and tumor vascularization $[15,16]$. Moreover, the number of studies evaluating the endoglin expression in several cancer types and targeting modulation of this expression by immunological interventions has been increasing recently [17].

Recent studies focus on novel diagnosis and treatment strategies for DN to decrease its mortality and morbidity. Therefore reliable markers for early detection and progression of DN are necessary along with molecular targets for personal treatment.

In this study we evaluated endocan and endoglin levels in normoalbuminuric diabetes and DN patients and compared them with healthy subjects. Therefore we aimed at gaining a better understanding of the role of angiogenesis in diabetes with these markers and to assess the predictive role of endocan and endoglin as markers of DN progression in DM patients.

\section{Material and methods}

The biochemical analyses were performed by the same researchers. The researcher performing ELISA analyses did not know which plasma belonged to which participant so he was blind.

\section{Ethical permission}

All procedures were in conformity with the ethical standards of the responsible committee on human experimentation (institutional and national) and with the Declaration of Helsinki. Informed consent was taken from all groups.

\section{Materials}

The glycated hemoglobin $\left(\mathrm{HbA}_{1 \mathrm{c}}\right)$ autoanalyser, Primus Premier Trinity Biotech-Hb9210 trademark, was from the USA; High-density lipoprotein cholesterol (HDL-C), total cholesterol, triglyceride (TG), fasting blood glucose (FBG), urinary albumin, total protein and creatinine were measured with Roche brand commercial kits in a Cobas e6000-e501 (Japan) device. Low-density lipoprotein cholesterol (LDL-C) was calculated by the Friedewald formula [18]. Sunred human endothelial cell-specific-molecule-1/endocan and endoglin ELISA commercial kits were purchased from PRC. A Bio Tek Microplate Readers (USA) brand ELISA device was used.

\section{Study design, eligibility criteria of subjects and analyses}

The participants were recruited from diabetes patients firstly admitted to our hospital and from 
healthy volunteers who were admitted to internal medicine out-patient clinics for a routine medical assessment. The control group was named group I. All participants were evaluated by medical history (including smoking status, medications used, hypertension history, duration of diabetes and oral antidiabetic drug or insulin usage), physical examination (weight, height, body mass index as kg/ $\mathrm{m}^{2}$, arterial blood pressure) and laboratory measurements $\left(\mathrm{HbA}_{1 \mathrm{c}}\right.$, fasting glucose, lipids, complete blood count, urea, creatinine, urinary daily albumin excretion). Subjects with clinical evidence of cardiovascular disease (coronary, peripheral, carotid artery), any other major disease (hepatic failure, end stage renal failure, any suspicious renal disease other than diabetic nephropathy such as glomerulonephritis, urolithiasis, any other disease causing nephrotic syndrome, etc., any malignancy, autoimmune diseases, acute or chronic infections), recent history of trauma or surgery, or pregnancy were excluded. 24-hour urinary albumin excretion of diabetic patients was measured twice in the absence of urinary tract infection (3 to 6 months apart). The normoalbuminuria (NA) upper limit was taken as $30 \mathrm{mg} /$ day of urinary albumin excretion [6]. The nephropathy group (DN) was defined as persistent albuminuria $\geq 30 \mathrm{mg} /$ day in two consecutive 24-hour urine analyses, normal or decreased glomerular filtration rate, the presence of diabetic retinopathy and no evidence of any other renal disease. Patients with no diabetic retinopathy changes in their fundus examination and accepted as having other etiologies of nephropathy were excluded from the study. The patients were classified as normoalbuminuric ( $<30 \mathrm{mg} /$ day of urinary albumin excretion) (named group II) or nephropathic (including microalbuminuria $=30-300 \mathrm{mg} /$ day and macroalbuminuria $>300 \mathrm{mg} /$ day) (named group III). End stage renal failure patients due to diabetic nephropathy were excluded from the study. Demographic data of the groups are shown in Table I. The healthy control group (group I, $n=35$ ) was age, sex and body mass index (BMI) matched with the DM groups (40 cases in group II and 56 cases in group III).

Blood samples were taken after a resting period of $15 \mathrm{~min}$ between 8:00 and 10:00 a.m. from the cannulated antecubital vein. Serum was prepared by centrifugation at $2000 \mathrm{~g}$ for $15 \mathrm{~min}$ at $+4^{\circ} \mathrm{C}$. Serum samples were stored at $-80^{\circ} \mathrm{C}$ until analysis.

Sensitivity of the endocan and endoglin commercial kits was $7.5 \mathrm{ng} / \mathrm{l}$ and $0.25 \mathrm{ng} / \mathrm{ml}$ while the

Table I. Demographic and laboratory data of the groups

\begin{tabular}{|c|c|c|c|}
\hline Parameter & $\begin{array}{c}\text { Group I } \\
\text { (healthy control) } \\
n=35\end{array}$ & $\begin{array}{c}\text { Group II } \\
\text { (normoalbuminuria) } \\
n=40\end{array}$ & $\begin{array}{c}\text { Group III } \\
\text { (nephropathy) } \\
n=56\end{array}$ \\
\hline Age [years] & $57.31 \pm 8.41$ & $56.36 \pm 8.55$ & $55.17 \pm 6.68$ \\
\hline Gender F/M & $17 / 18$ & $18 / 22$ & $26 / 30$ \\
\hline Height $[\mathrm{cm}]$ & $163.4 \pm 10.2$ & $161.2 \pm 8.1$ & $161.19 \pm 8.8$ \\
\hline Weight [kg] & $79.4 \pm 16.5$ & $85.7 \pm 14.7$ & $82.4 \pm 13.5$ \\
\hline Duration of diabetes [years] ${ }^{\star}$ & - & $5.57(1-26)$ & $10.6(1-33)$ \\
\hline $\mathrm{SBP}[\mathrm{mm} \mathrm{Hg}]$ & $119 \pm 4.52$ & $129.7 \pm 18.0$ & $131.4 \pm 14.9$ \\
\hline $\mathrm{DBP}[\mathrm{mm} \mathrm{Hg}]$ & $75 \pm 2.25$ & $77.2 \pm 11.0$ & $76.8 \pm 9.7$ \\
\hline $\mathrm{FBG}[\mathrm{mg} / \mathrm{dl}]^{*}$ & $92.3(80-96)$ & $131.5(80-371)$ & $182(96-432)$ \\
\hline $\mathrm{HbA}_{1 \mathrm{c}}(\%)^{*}$ & $4.7(4.3-5.3)$ & $7.3(5.6-17.2)$ & $8.4(6-14.4)$ \\
\hline $\operatorname{LDL}[\mathrm{mg} / \mathrm{dl}]^{*}$ & $121(81-146)$ & $136.1(76-220)$ & $129.4(66-291)$ \\
\hline $\mathrm{TG}[\mathrm{mg} / \mathrm{dll}]^{\star}$ & $125.0(50-158)$ & $170.9(36-466)$ & $185.6(53-623)$ \\
\hline $\mathrm{HDL}[\mathrm{mg} / \mathrm{dl}]$ & $53.4 \pm 16.41$ & $46.3 \pm 14.5$ & $44.6 \pm 14.2$ \\
\hline Urea [mg/dl] & $27.0 \pm 7.6$ & $29.8 \pm 7.1$ & $35.8 \pm 11.8$ \\
\hline $\mathrm{Cr}[\mathrm{mg} / \mathrm{dl}]$ & $0.70 \pm 0.11$ & $0.83 \pm 0.13$ & $0.91 \pm 0.19$ \\
\hline Endocan $[\mathrm{ng} / \mathrm{l}]^{*}$ & $680.77^{\mathrm{a}, \mathrm{c}}(213.3-1433.1)$ & $1011.4^{\mathrm{a}, \mathrm{b}}(429.9-1681.8)$ & $1175.3^{b, c}(564.5-1637.5)$ \\
\hline Endoglin $[\mathrm{ng} / \mathrm{ml}]^{*}$ & $20.58(11.06-58.85)$ & $20.96^{\mathrm{d}}(12.0-34.86)$ & $24.05^{d}(10.0-53.0)$ \\
\hline
\end{tabular}

${ }^{a}$ Control - Normoalbuminuria: $p=0.001$, ${ }^{b}$ Normoalbuminuria - Nephropathy: $p=0.011$, ${ }^{c}$ Control - Nephropathy: $p<0.001$, ${ }^{d}$ Normoalbuminuria - Nephropathy: $p=0.822$. Unless otherwise noted, data are presented as mean \pm standard deviation, ${ }^{*}$ median (min.-max.); SBP - systolic blood pressure, DBP - diastolic blood pressure, FBG - fasting blood glucose, HbA $A_{1 c}-$ glycated hemoglobin, $T G$ - triglycerides, $H D L$ - high-density lipoprotein, $L D L$ - low-density lipoprotein, $\mathrm{Cr}$-creatinine. 
intra-assay coefficient of variation $(C V)<10 \%$; inter assay $\mathrm{CV}<12 \%$. Assay ranges of endocan and endoglin were $7.5-2000 \mathrm{ng} / \mathrm{l}$ and $0.25-70 \mathrm{ng} / \mathrm{ml}$ respectively.

\section{Statistical analysis}

Statistical analysis was performed with SPSS version 17 (Chicago, Illinois). Homogeneity of groups' data was analyzed with the Shapiro-Wilk test. Results are shown as a mean \pm standard deviation or median (min.-max.) depending on data distribution. Normally distributed data were analyzed using the independent-samples $t$ test and one-way ANOVA. Abnormally distributed data were analyzed with the Mann-Whitney $U$ and Kruskal-Wallis tests. The Pearson test was used for correlation analysis. Alpha significance level was set as $<0.05$.

\section{Results}

In comparison of the DN group (group III) with the NA diabetes group (group II), fasting blood glucose, $\mathrm{HbA}_{1 \mathrm{c}}$, urea levels and duration of diabetes in DN patients were higher than in NA patients $(p<0.001, p<0.001 p=0.025, p<0.001$ respectively). Other parameters' differences did not reach statistical significance $(p>0.05)$. Endocan levels of DN patients were higher than those of NA patients $(p=0.011)$. Endoglin levels of DN patients were higher than those of NA patients also but this difference did not reach statistical significance $(p=0.822)$.

In comparison of the DN group (group III) with the healthy control group (group I), endocan and endoglin levels of the DN group were higher than the control group, reaching statistical significance ( $p<0.001$ and $p=0.002$ respectively).

In comparison of the NA group (group II) with healthy controls (group I), there were statistically significant differences in terms of endocan and endoglin levels as higher levels in the NA group ( $p=0.001$ and $p=0.017$ respectively).

The differences of groups in terms of endocan and endoglin levels are shown in Figures 1 and 2.

In receiver operating characteristic (ROC) curve analysis, the cut-off value for serum endocan was $1070 \mathrm{ng} / \mathrm{l}$ with a sensitivity of $68.3 \%$ and a specificity of $57.5 \%$ in determining DN.

When endocan's correlations with other parameters in only the DN group were assessed, a positive correlation was found only with endoglin and $\mathrm{HbA}_{1 \mathrm{c}}$ levels $(r=0.512, p<0.001$ and $r=$ $0.262, p=0.043$ respectively) (Figure 3 ).

When endoglin's correlations with other parameters in only the DN group were assessed, a positive correlation was found only with $\mathrm{HDL}$ cholesterol, with a $p$-value of 0.016 and $r$ value of
0.312. The correlation of endoglin with $\mathrm{HbA}_{1 \mathrm{c}}$ did not reach statistical significance $(p=0.781)$.

\section{Discussion}

Nephropathy is one of the major microvascular complications of DM and in the industrialized

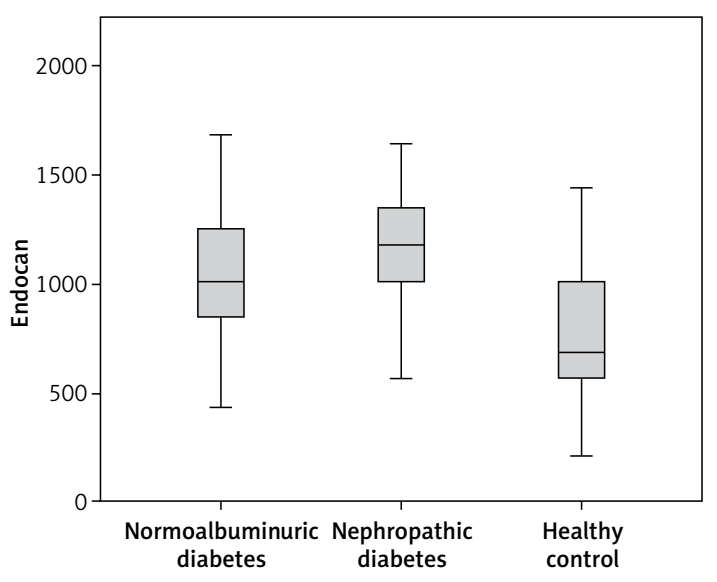

Figure 1. Box-plot graphic of endocan levels of the study population

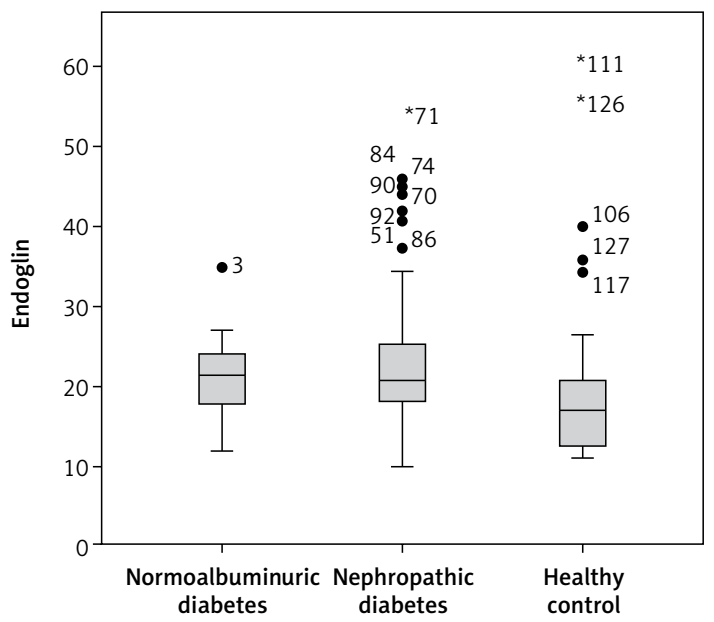

Figure 2. Box-plot graphic of endoglin levels of the study population

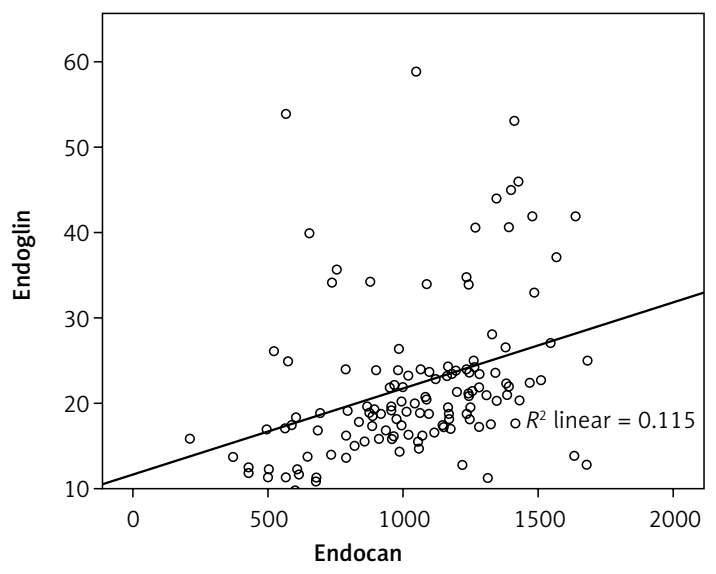

Figure 3. Scattered-dot graphic of endocan and endoglin correlation 
world the morbidity and mortality due to DN is continually increasing. No adequate exact therapeutic option is currently available for the treatment of patients with nephropathy [19].

The discovery of novel markers of pathophysiology is very important in preventing development of diabetes mellitus and its complications, identifying diabetes patients at high risk for incident DN, as well as in providing targets for therapy. In recent years our knowledge about the etiology of diabetes mellitus and its vascular complications has widened noticeably. So the search for markers of diabetic vascular complications continues [20]. Many cytokines modulating different steps in pathophysiology of diabetes have come into consideration and so many trials about these modulators have been published recently.

Arman et al. reported that serum endocan concentration was found to be independently correlated with urinary albumin to creatinine ratio (UACR); moreover UACR and endocan levels of diabetes patients without nephropathy declined with improvement of glycemic control [21].

In a study of Pawlak et al., CKD patients with or without cardiovascular disease (CVD) were evaluated and compared with a healthy control group in terms of some vascular adhesion molecules (sICAM-1, sVCAM-1), inflammatory markers (hsCRP, IL-6, TNF- $\alpha$ ) and endocan levels. In this study, endocan levels of CKD patients were higher than in the controls, and moreover endocan levels of CKD patients with CVD were higher than in their counterparts without CVD [22]. Like this study, in our study also diabetic nephropathy patients' endocan levels were higher than those of the control group.

Endocan levels of 251 CKD patients and 60 non-diabetic non-CKD controls were evaluated in a study of Yilmaz et al. and endocan levels of CKD patients were higher than those of the control group [23]. The CKD group included 58 diabetes patients and in the analysis, higher endocan levels were recorded in diabetes patients than non-diabetic CKD patients and the control group. In our study also, we found a statistically significant difference in terms of endocan levels between diabetes patients and the healthy control group like this study. But in the study of Yilmaz et al., the control group was constituted from non-diabetics, people and so did not include any diabetes patients without CKD. For this reason no information regarding the difference between nephropathic and non-nephropathic diabetes was obtained.

In a study evaluating the value of soluble endoglin as an indicator of cardiovascular alterations in hypertensive and diabetes patients, $\mathrm{HbA}_{1 c}$-endoglin correlation did not reach statistical significance in diabetes patients with or without hypertension.
In our study's diabetes group, $\mathrm{HbA}_{1 c}$-endoglin correlation did not reach statistical significance also. But when this correlation was assessed in diabetes patients as a whole group in Blazquez-Medela's study, a statistically significant positive correlation was found between soluble endoglin and glycated hemoglobin levels [24].

In another study examining vitreous sample endocan and soluble endoglin levels of proliferative diabetic retinopathy patients and non-diabetic controls, endocan and soluble endoglin levels in retinopathy patients were found to be higher than in non-diabetics [25]. Diabetic retinopathy and nephropathy have a similar pathogenetic basis in terms of aberrant angiogenesis. Our study examining serum levels of endoglin in another microvascular complication of diabetes mellitus gave similar results in terms of higher endoglin levels in diabetes patients with microvascular complication. So the results of our study might support the conclusion that both microvascular complications could share a similar pathogenetic cascade in terms of angiogenesis.

The early recognition of patients at high risk for development of diabetic nephropathy and early diagnosis of this frequent complication might protect the patients from being a candidate for renal replacement therapies and decrease the health burden of this time-, life-, and money-consuming complication. So the results of our study planned to evaluate these two bio-markers' levels at different stages of diabetic nephropathy might be used in clinical practice to identify high-risk patients and treat them more aggressively to defer the development and progression of this complication.

There are some limitations that should be considered in the present study. First of all, we assessed the circulating serum levels of endocan and endoglin in diabetic nephropathy patients. If we could study the renal tissue levels of them in diabetic and non-diabetic subjects, some different results might be obtained. Secondly, our sample size was relatively small due to our financial limitations, and it was a cross-sectional, single-center study. Lastly, it was not possible for us to homogenize the study group according to their medication groups such as diabetes therapy (different oral antidiabetic groups or insulin), antihypertensive (renin-angiotensin-aldosterone system blockers, $\beta$-blockers, calcium channel blockers, diuretic groups, etc.), anti-lipemic and anti-aggregant medication groups, which might give unreliable results due to our small sample size. Evaluating these details in larger studies in the future might clarify the effect of some medication groups on these two biomarkers also. Larger sample sized, long-term, prospective and multicenter-designed new studies are needed to confirm these results. 
In conclusion, the complications of DM are still the leading causes of morbidity and mortality in DM. Understanding the pathogenetic mechanisms of complications and early recognition of complication development by some new markers might add some benefit in inhibition of progression of complications and in development of new treatment strategies on a molecular basis. In our study, we suggested that endocan might be a more reliable new marker of diabetic nephropathy development than endoglin. But this issue must be investigated with new, larger, multi-center prospective studies.

\section{Conflict of interest}

The authors declared no conflict of interest.

\section{References}

1. Montero RM, Covic A, Gnudi L, Goldsmith D. Diabetic nephropathy: what does the future hold? Int Urol Nephrol 2016; 48: 99-113.

2. Checheriță I, Manda G, Hinescu ME, et al. New molecular insights in diabetic nephropathy. Int Urol Nephrol 2016; 48: 373-87.

3. Bakris GL. Overview of diabetic nephropathy. UpToDate. http://www.uptodate.com/contents/overview-of-diabeticnephropathy.com. Accessed Nov 2015.

4. Ritz E. Pathogenesis, clinical manifestations, and natural history of diabetic nephropathy. In: Comprehensive Clinical Nephrology. $3^{\text {rd }}$ ed. Feehally J, Floege J, Johnson RJ (eds.). Mosby Elsevier, Philadelphia 2007; 353-64.

5. Mogensen CE, Christensen CK, Vittinghus E. The stages in diabetic renal disease: with emphasis on the stage of incipient diabetic nephropathy. Diabetes 1983; 32: 64-78.

6. Mogensen CE, Chachati A, Christensen CK, et al. Microalbuminuria: an early marker of renal involvement in diabetes. Uremia Invest 1985-1986; 9: 85-95.

7. Martin A, Komada MR, Sane DC. Abnormal angiogenesis in diabetes mellitus. Med Res Rev 2003; 23: 117-45.

8. Zent R, Pozzi A. Angiogenesis in diabetic nephropathy. Semin Nephrol 2007; 27: 161-71.

9. Sarrazin S, Adam E, Lyon M, et al. Endocan or endothelial cell specific molecule-1 (ESM-1): a potential nove endothelial cell marker and a new target for cancer therapy. Biochim Biophys Acta 2006; 1765: 25-37.

10. Kali A, Shetty KS. Endocan: a novel circulating proteoglycan. Indian J Pharmacol 2014; 46: 579-83.

11. Lassalle P, Molet $S$, Janin A, et al. ESM-1 is a novel human endothelial cell-specific molecule expressed in lung and regulated by cytokines. J Biol Chem 1996; 271: 20458-64.

12. Balta S, Mikhailidis DP, Demirkol S, Ozturk C, Celik T, Iyisoy $\mathrm{A}$. Endocan: a novel inflammatory indicator in cardiovascular disease? Atherosclerosis 2015; 243: 339-43.

13. Pérez-Gómez E, Del Castillo G, Juan Francisco S, LópezNovoa JM, Bernabéu C, Quintanilla M. The role of the TGF-beta coreceptor endoglin in cancer. Sci World I 2010; 10: 2367-84.

14. Conley BA, Smith JD, Guerrero-Esteo M, Bernabeu C, Vary CP. Endoglin, a TGF-beta receptor-associated protein, is expressed by smooth muscle cells in human atherosclerotic plaques. Atherosclerosis 2000; 153: 323-35.
15. Jonker L, Arthur HM. Endoglin expression in early development is associated with vasculogenesis and angiogenesis. Mech Dev 2002; 110: 193-6.

16. Jang YS, Choi IH. Contrasting roles of different endoglin forms in atherosclerosis. Immune Netw 2014; 14: 237-40.

17. Peker KD, Ozkanli SS, Akyuz C, et al. Preoperative immunonutrition regulates tumor infiltrative lymphocytes and increases tumor angiogenesis in gastric cancer patients. Arch Med Sci 2017; 13: 1365-72.

18. Friedewald WT, Levy RI, Fredrickson DS. Estimation of the concentration of low-density lipoprotein cholesterol in plasma, without use of the preparative ultracentrifuge. Clin Chem 1972; 18: 499-502.

19. Balakumar P, Arora MK, Ganti SS, Reddy J, Singh M. Recent advances in pharmacotherapy for diabetic nephropathy: current perspectives and future directions. Pharmacol Res 2009; 60: 24-32.

20. Kahraman C, Kahraman NK, Aras B, et al. The relationship between neutrophil-to-lymphocyte ratio and albuminuria in type 2 diabetic patients: a pilot study. Arch Med Sci 2016; 12: 571-5.

21. Arman Y, Akpinar TS, Kose M, et al. Effect of glycemic regulation on endocan levels in patients with diabetes: a preliminary study. Angiology 2016; 67: 239-44.

22. Pawlak K, Mysliwiec M, Pawlak D. Endocan - the new endothelial activation marker independently associated with soluble endothelial adhesion molecules in uraemic patients with cardiovascular disease. Clin Biochem 2015; 48: 425-30

23. Yilmaz MI, Siriopol D, Saglam M, et al. Plasma endocan levels associate with inflammation, vascular abnormalities, cardiovascular events, and survival in chronic kidney disease. Kidney Int 2014; 86: 1213-20.

24. Blázquez-Medela AM, García-Ortiz L, Gómez-Marcos MA, et al. Increased plasma soluble endoglin levels as an indicator of cardiovascular alterations in hypertensive and diabetic patients. BMC Med 2010; 8: 86.

25. Abu El-Asrar AM, Nawaz MI, De Hertogh G, et al. The angiogenic biomarker endocan is upregulated in proliferative diabetic retinopathy and correlates with vascular endothelial growth factor. Curr Eye Res 2015; 40: 321-31. 